\title{
Minimalinvasive Osteosynthese der Skaphoidfraktur
}

\author{
Benjamin Ziegler, Berthold Bickert, Ulrich Kneser
}

\section{Zusammenfassung}

Skaphoidfrakturen stellen schwere Verletzungen der Handwurzel dar. Dennoch werden sie im Rahmen der ersten ärztlichen Vorstellung nach Trauma häufig nicht diagnostiziert und erst verspätet therapiert, was zu einer erheblichen Verschlechterung der Heilungschancen und damit zu fatalen Langzeitfolgen führen kann. Skaphoidfrakturen stellen daher hohe Anforderungen an die sichere Diagnostik und eine konsequente Therapie. Stabile Frakturen können sowohl konservativ als auch operativ versorgt werden, während alle instabilen Frakturen einer operativen Stabilisierung bedürfen. Diese kann heute in vielen Fällen durch minimalinvasive Zugänge mit geringem Operationstrauma, kurzer Hospitalisierung und frühfunktioneller Nachbehandlung erfolgen. Frühestens 6 Wochen nach Versorgung kann mit dem Belastungsaufbau begonnen werden.

\section{Minimally Invasive Treatment for Scaphoid Fractures}

Fractures of the scaphoid are severe injuries of the carpal joint. Nevertheless, correct therapy is often delayed due to difficulties in the early diagnosis resulting in significantly worsening of the chances of recovery and a disastrous long-term disability. Therefore scaphoid fractures demand reliable diagnostics and a consistent therapy. While stable fractures can be treated by immobilisation as well as by osteosynthesis, unstable fractures always need an operative stabilisation. Today, this can be often achieved by a minimally invasive approach with the benefit of minimised surgical trauma, short hospital stay and an early functional postoperative treatment. Increased load bearing is allowed at the earliest six weeks after operative treatment.

\section{Einleitung}

Skaphoidfrakturen stellen schwere Verletzungen der Handwurzel infolge starker, äußerer Krafteinwirkung dar. Frühzeitig erkannt und verletzungsgerecht therapiert können sie vollständig ausheilen und weisen eine gute Prognose auf. Bei verzögerter Diagnose oder unzureichender Therapie besteht jedoch ein erhebliches Risiko für bleibende Schäden durch Ausbildung einer Skaphoidpseudarthrose und konsekutiv entstehender Arthrose des Handgelenks (sog. SNACArthrose). Führt diese schließlich zu einem fortgeschrittenen karpalen Kol-

OP-JOURNAL 2015; 31: 104-107

(c) Georg Thieme Verlag KG Stuttgart · New York DOI http://dx.doi.org/10.1055/s-0035-1546003

laps, resultiert nicht selten neben einer Einschränkung der Lebensqualität im Alltag auch der Verlust der Arbeitsfähigkeit. Um sowohl volkswirtschaftliche als auch individuelle Folgen der Verletzungen zu minimieren, sind eine zuverlässige Diagnostik wie auch die sichere Therapie der Skaphoidfraktur unabdingbar. Während für bestimmte Formen der Skaphoidfraktur die konservative Therapie weiterhin ihren berechtigten Stellenwert hat, zeigte die Osteosynthese des Skaphoids in den letzten 30 Jahren eine rasante Entwicklung. Heute stehen gleichermaßen effektive wie schonende Möglichkeiten der minimalinvasiven Osteosynthese des Skaphoids zu Verfügung, welche in der Indikationsstellung zur konservativen vs. operativen Therapie ihre Berücksichtigung finden sollten.

\section{Pathomechanismen}

Die Skaphoidfraktur ist eine insgesamt seltene Verletzung. Sie stellt jedoch mit etwa $80 \%$ die bei Weitem häufigste Fraktur der Handwurzel dar. Der Altersgipfel liegt zwischen 20 und 40 Jahren, wobei Männer etwa 6-mal häufiger betroffen sind als Frauen. Bei Kindern sind Skaphoidfrakturen aufgrund der noch unvollständig ausgebildeten Handwurzel selten, bei älteren Menschen verschiebt sich die Häufigkeit der Fraktur bei vergleichbarer Krafteinwirkung hin zur distalen Radiusfraktur. 60-80\% der Skaphoidfrakturen betreffen das mittlere Drittel des Handwurzelknochens, während sich die verbleibenden Frakturen zu etwa gleichen Teilen auf das proximale und das distale Drittel aufteilen [15].

Ursache der Fraktur ist in den meisten Fällen ein Sturz auf die dorsalextendierte Hand. Die dabei resultierende, plötzliche Hyperextension des Karpus führt zu einer Überlastung der palmaren Kortikalis des Skaphoids, welche darunter frakturiert. Seltener bedingt eine direkte Gewalteinwirkung durch Quetschung oder Anprall (früher der Kurbelrückschlag) eine Fraktur des Skaphoids [13].

\section{Diagnostik}

Leitsymptom der Verletzung ist der radiokarpal betonte Druck- und Bewegungsschmerz. Er lässt sich v.a. durch Druck auf die Tabatière sowie durch Hyperextension und Radialduktion des Handgelenks auslösen. Darüber hinaus findet sich häufig ein Stauchungsschmerz des 1., manchmal auch des 2. Strahles der Hand.

An 1. Stelle der weiteren apparativen Diagnostik stehen die konventionellen Röntgenaufnahmen des Handgelenks. Hierbei sollten die Standardaufnahmen (s. Abb. 1) mit postero-anteriorem (pa) sowie streng seitlichem Strahlengang routinemäßig durch Spezialaufnahmen 

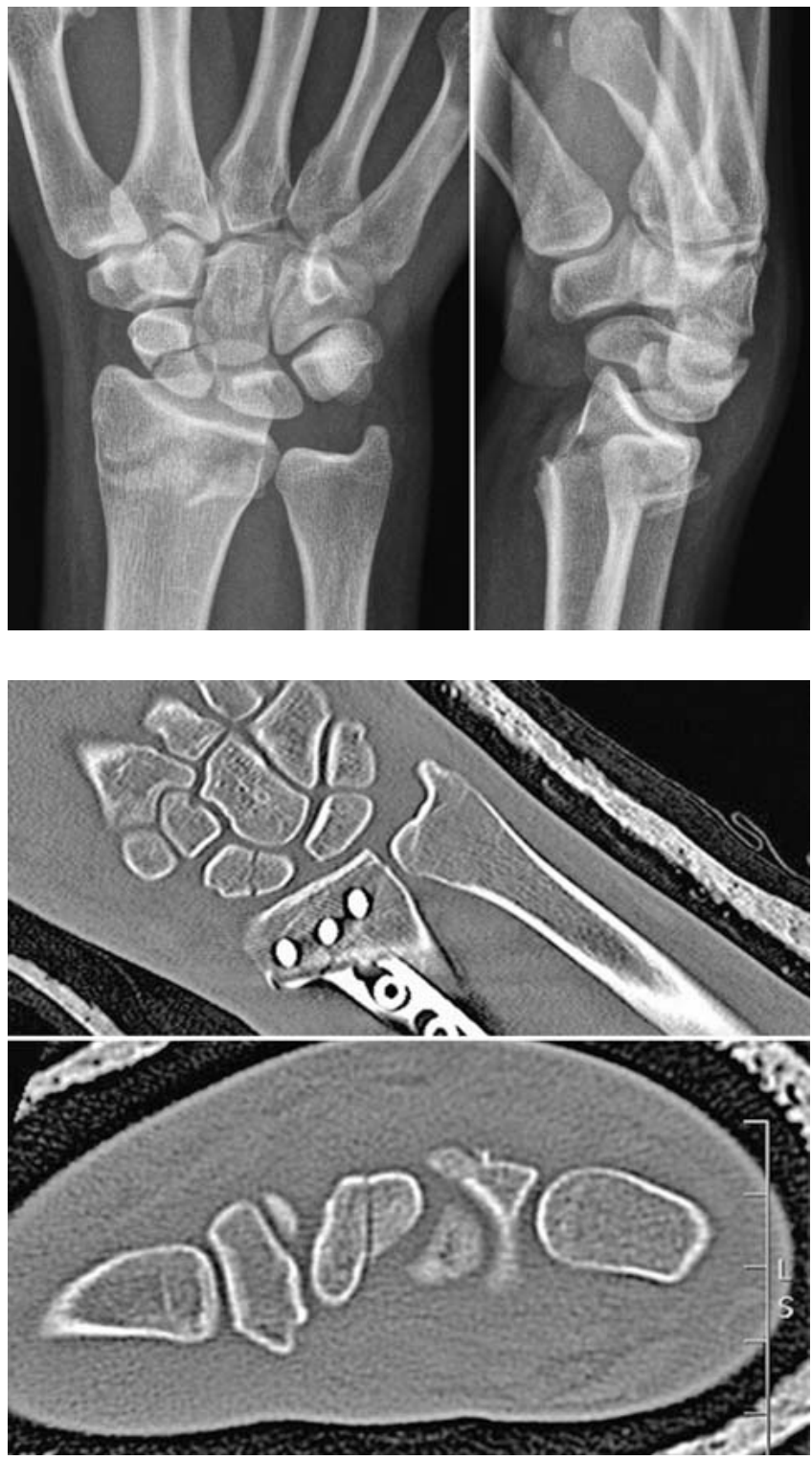

Abb. 2 Nach primärer Osteosynthese der Radiusfraktur erfolgt die weitere diagnostische Abklärung der Skaphoidfraktur mittels CT. Die standardmäßige koronare Rekonstruktion der Handwurzel (oben) genügt nicht. Erst die obligate Rekonstruktion in der Längsebene des Skaphoids (unten) vermittelt einen korrekten Eindruck des Frakturverlaufs. ergänzt werden. Die Aufnahme nach Stecher, bei welcher die zur Faust geschlossene Hand in Ulnarduktion gehalten wird, erlaubt dabei eine überlagerungsfreie Darstellung des Skaphoids in seiner Längsachse [14]. Weitere Spezialaufnahmen des sog. Skaphoidquartetts haben heute zugunsten der Schnittbildverfahren an Bedeutung verloren.

Wurde durch klinische Untersuchung und Röntgendiagnostik der Verdacht auf eine Skaphoidfraktur gestellt, ist die weitere Diagnostik durch Computertomografie (CT) obligat.

Hierbei sollte für die korrekte Diagnose der Fraktur und die weitere Therapieplanung explizit die sagittale Längsebene des Skaphoids mit einer maximalen Schichtdicke von $1 \mathrm{~mm}$ dargestellt werden (Abb. 2).

\section{Klassifikation der Skaphoidfraktur}

Ausgehend von der radiologischen Diagnostik erfolgt die im klinischen Alltag gebräuchlichste Klassifizierung der Skaphoidfraktur nach Herbert. Diese teilt die Frakturen in die stabilen Frakturformen Tuberkelfraktur (A1) und inkomplette Taillenfraktur (A2) sowie in die instabilen Schrägfrakturen (B1), dislozierten Frakturen der Skaphoidtaille (B2), die immer hochgradig instabilen Frakturen des proximalen Pols (B3) und die transskaphoidalen Luxationsfrakturen (B4) ein [5]. Mit routinemäßiger CTDiagnostik in korrekter Schnittführung konnte gezeigt werden, dass inkomplette Frakturen beim Erwachsenen nicht vorkommen, sodass die A2-Fraktur als unverschobene Fraktur der Skaphoidtaille neu definiert wurde [9].

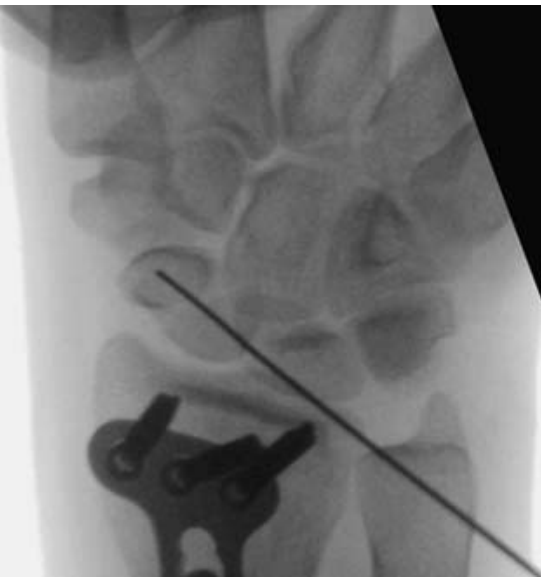

Abb. 3 Zur minimalinvasiven Frakturversorgung wird zunächst ein Kirschner-Draht perkutan von dorsal in den proximalen Skaphoidpol eingebracht und unter Bildwandler-Kontrolle vorgeschoben.

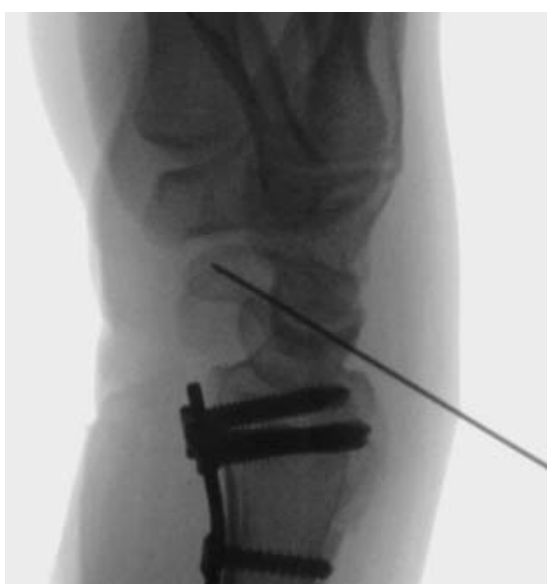

Abb. 4 Die korrekte Lage wird zunächst in zweiter Ebene sowie zusätzlich durch vollständiges Drehen des Unterarms unter dem Bildwandler kontrolliert.

\section{Konservative Therapie}

Eine konservative Therapie ist für stabile, unverschobene Frakturen des mittleren und distalen Drittels indiziert, also für Frakturen vom Typ A1 und A2 nach Herbert-Krimmer. Insbesondere die A2Fraktur kann nur im CT sicher diagnostiziert werden, sodass die CT-Diagnostik als Voraussetzung für eine konservative Therapie anzusehen ist. Die Immobilisation erfolgt im Unterarm-Cast für mindestens 6 Wochen. Die Notwendigkeit des Daumeneinschlusses war lange umstritten $[1,4,8]$. Nach neueren Ergebnissen ist der Einschluss des 1. Mittelhandstrahls und Freilassen des Daumengrundgelenks ausreichend [3]. Ein Oberarmgips ist zur Immobilisation des Skaphoids nicht erforderlich und wegen seiner negativen Auswirkungen auf die Ellbogenfunktion seit Längerem obsolet. 


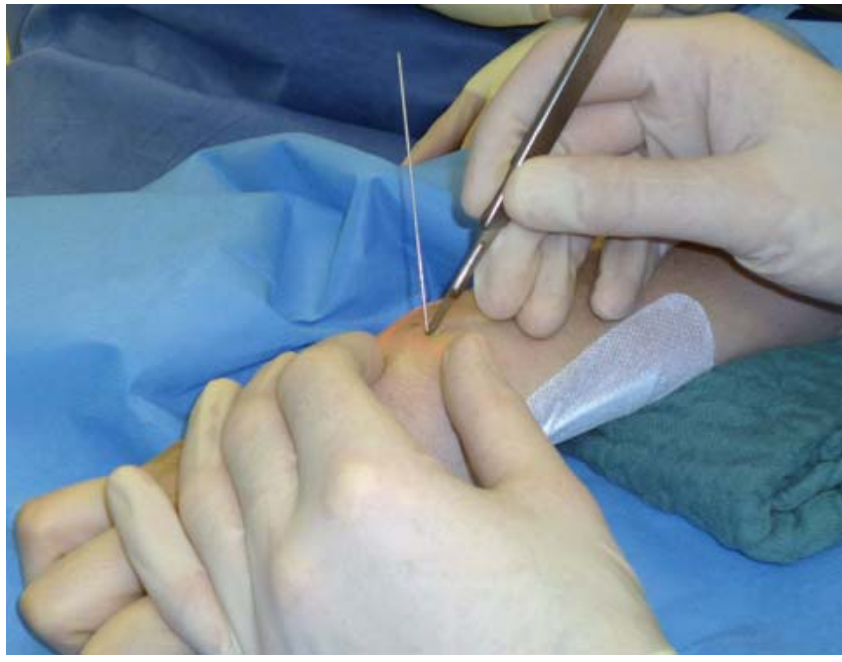

Abb. 5 Nun etwa $0,5 \mathrm{~cm}$ messender Hautschnitt unmittelbar am einliegenden Kirschner-Draht.

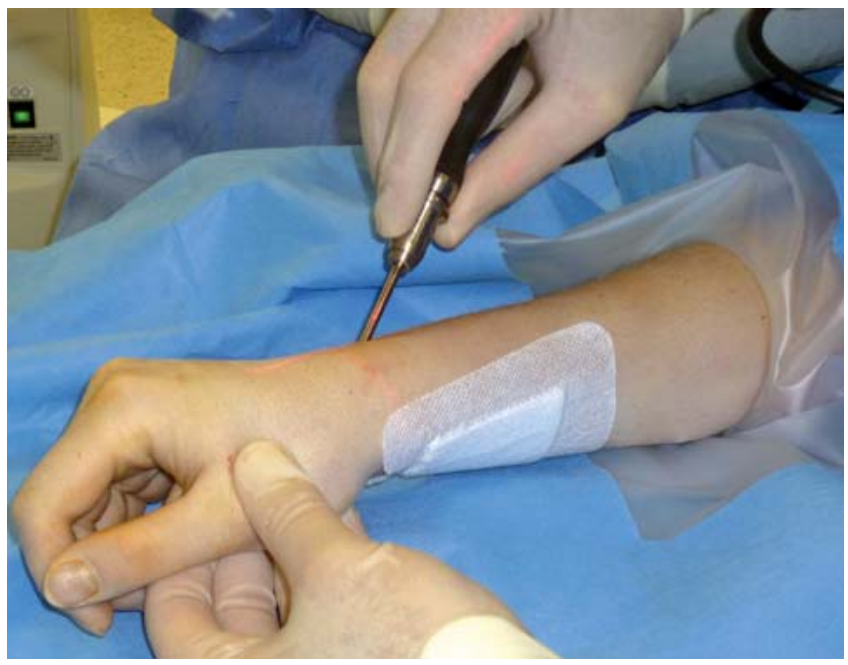

Abb. 6 Einbringen der selbstschneidenden, kanülierten Mini-Doppelgewindeschraube über den Führungsdraht.
Nach 6 Wochen erfolgen die Abnahme des Skaphoid-Casts und die radiologische Kontrolle, unbedingt mit StecherAufnahme. Ist eine ausreichende Konsolidierung der Fraktur nicht sicher nachweisbar, ist die Kontrolle der Frakturheilung durch CT indiziert.

\section{Operative Therapie}

Obgleich stabile Frakturen (Typ A2) durch konservative Therapie in den meisten Fällen zur Ausheilung gebracht werden können, wird auch für diese Frakturen die primäre Versorgung durch Osteosynthese diskutiert. Sie ermöglicht den Verzicht auf eine langwierige Ruhigstellung und den frühzeitigen Beginn einer funktionellen Beübung. Zahlreiche Studien beobachteten eine schnellere Konsolidierung der Fraktur sowie eine frühere Wiederaufnahme der beruflichen Tätigkeit, insbesondere für körperlich arbeitende Patienten, unter der Inkaufnahme des Risikos eines operativen Eingriffs $[2,10,11]$.

Instabile Frakturen (Typ B nach Herbert) sollten immer einer operativen Therapie zugeführt werden, da sonst ein hohes Risiko für eine Pseudarthrose besteht.

Die Osteosynthese erfolgt dabei in der überwiegenden Mehrzahl durch das intraossäre Einbringen einer Doppelgewindeschraube, wie sie 1984 von Herbert vorgestellt wurde. Die Herbert-Schraube verfügt über 2 unterschiedlich steile Gewinde an proximalem und distalem Schraubenpol, wodurch bei Einbringung der Schraube in das Skaphoid eine Kompression des Frakturspalts erreicht wird. Durch die vollständig intraossäre Lage der Schraube ist keine Metallentfernung notwendig und die Beeinträchtigung der Gelenkflächen minimal [5]. Zahlreiche Modifikationen der Herbert-Schraube in den letzten 15 Jahren verbesserten die Schraubengriffigkeit und ermöglichten durch Kanülierung ein komfortables Einbringen über Führungsdraht selbst für Schrauben von nur 2,2 mm Durchmesser.

Die Wahl des Operationsverfahrens und des Zugangs orientiert sich am Verlauf und der Lage der Fraktur im Skaphoid (proximales Drittel vs. Fraktur des mittleren oder distalen Drittels) sowie am Grad der Dislokation.

Liegt die Fraktur im proximalen Drittel, wird die Schraube über einen dorsalen Zugang vom proximalen Pol des Knochens aus antegrad eingebracht. Frakturen des distalen Drittels werden von palmar versorgt, die Doppelgewindeschraube wird hier retrograd vom distalen Pol in das Skaphoid eingebracht. Wenn die Fraktur im mittleren Drittel durch die Taille des Skaphoids zieht, ist für keinen der beiden Zugänge eine Überlegenheit erwiesen. Aufgrund des geringeren Risikos der palmaren Abkippung des proximalen Fragments und der einfacheren radiologischen Lagekontrolle, empfehlen einige Autoren die Verschraubung von palmar [8]. Im retrospektiven Vergleich konnte jedoch keine Überlegenheit in Frakturheilung und Funktion gegenüber einem dorsalen $\mathrm{Zu}$ gang nachgewiesen werden [7]. Falls also die Frakturebene im mittleren Drittel schräg liegt, sodass eine palmare retrograde Schraube fast parallel zur Fraktur laufen würde, ist oft der dorsale Zugang vorzuziehen, wenn mit diesem die Frak- tur näher an der Senkrechten zur Frakturebene überbrückt werden kann.

\section{Minimalinvasive Skaphoidosteo- synthese retrograd von palmar}

Gelingt die geschlossene Reposition der Fraktur bzw. eine perkutane Reposition über sog. Joystick-Drähte oder liegt eine undislozierte Fraktur vor, kann die Osteosynthese mittels kanülierter Doppelgewindeschraube minimalinvasiv erfolgen.

Für den palmaren, minimalinvasiven $\mathrm{Zu}-$ gang wird zunächst die Lage des STT-Gelenks unter Bildwandler-Durchleuchtung (BV) mit einem Draht aufgesucht. Im Anschluss erfolgt hier ein limitierter Hautschnitt von unter $1 \mathrm{~cm}$ Länge. Nach maximaler Dorsalextension des Handgelenks über einem geeigneten Bänkchen wird mit der Bohrdrahthülse der Kontakt zum Skaphoid gesucht und der Führungsdraht der Schraube unter Durchleuchtungskontrolle in den proximalen Skaphoidpol vorgeschoben. Die Lagekontrolle des Drahtes erfolgt in den 3 Standardebenen des Skaphoids (pa, seitlich und Stecher) sowie mit Durchleuchtung unter Rotation, durch die ein sehr guter 3-D-Eindruck der Reposition und der Platzierung des Drahtes resultiert. Erst bei perfekter Lage des Drahtes wird die Schraube eingedreht. Die erforderliche Schraubenlänge ist manchmal nur unzuverlässig zu messen. Eine typische Länge beim Erwachsenen sind $22 \mathrm{~mm}$, bei optimal-zentraler Platzierung im Skaphoid bis $24 \mathrm{~mm}$, bei kürzerem Diameter bis $20 \mathrm{~mm}$. Nach Lagekontrolle der Schraube wird der Draht entfernt und die abschließende Röntgenkontrolle mit dem BV-Gerät durch- 


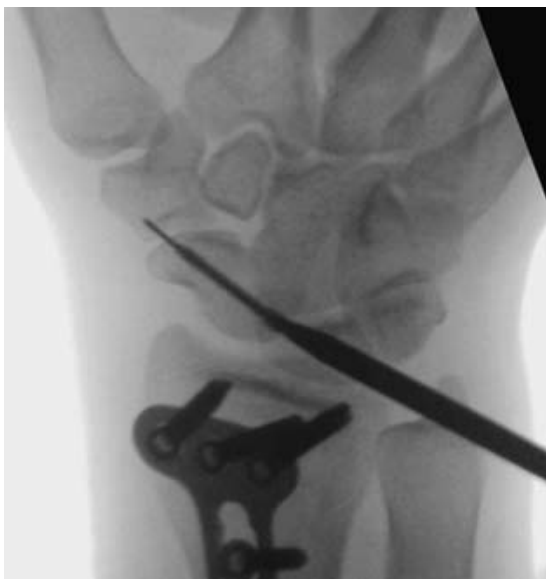

Abb. 7 Eindrehen der Schraube bis zur korrekten Lage unter Bildwandler-Kontrolle.

geführt. Wiederum werden die 3 Ebenen (pa, lateral und Stecher) eingestellt und zusätzlich eine Durchleuchtung mit Unterarmrotation durchgeführt. Hierbei wird v.a. darauf geachtet, dass der Frakturspalt adäquat von der Schraube überbrückt ist und dass weder das proximale noch das distale Ende der Schraube ins Gelenk ragen $[6,12]$.

\section{Minimalinvasive Skaphoidosteo- synthese antegrad von dorsal}

Für den dorsalen, minimalinvasiven $\mathrm{Zu}-$ gang wird unter BV-Durchleuchtung der proximale Skaphoidpol am Dreieck zwischen radioskaphoidalem und skapholunärem Gelenkspalt mit einem Führungsdraht der geplanten Mini-Doppelgewindeschraube aufgesucht. Die Eintrittsstelle in die Haut liegt dabei immer überraschend weit ulnar. Der Draht wird ins Skaphoid vorgebohrt, seine Lage wird wieder mit BV in den 3 Standardebenen des Skaphoids und unter Durchleuchtung bei Unterarmrotation kontrolliert. Bei korrekter Lage wird die Haut um den Draht herum auf etwa $0,5 \mathrm{~cm}$ inzidiert und dann eine selbstschneidende Mini-Doppelgewindeschraube eingedreht. Beim Eindrehen wird eine Berührung der Schraube mit der Haut sorgfältig vermieden. Die Schraubenlänge lässt sich hier nicht messen, sondern nur schätzen, wobei die Schraube in der distalen Spongiosa immer so gut hält, dass sie eher etwas zu kurz als zu lang gewählt werden sollte. Üblich ist eine Schraubenlänge zwischen 20 und $22 \mathrm{~mm}$. Die Abb. 1 bis 8 zeigen ein Fallbeispiel einer minimalinvasiven Skaphoidosteosynthese von dorsal mit Ausschnitten der präoperativen Diagnostik, Aufnahmen der operativen Versorgung und postoperativer Kontrolle.
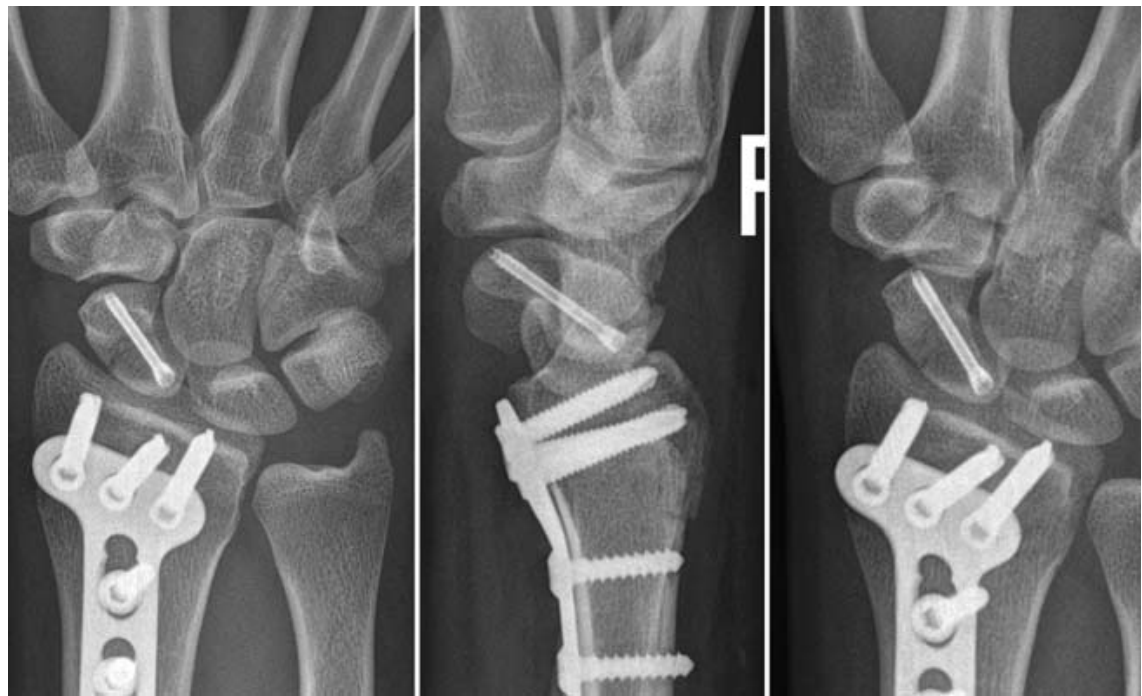

Abb. 8 In der postoperativen Röntgenkontrolle wird die postero-anteriore (links) und die lateral Aufnahme (Mitte) ergänzt durch die Aufnahme nach Stecher (rechts).

\section{Postoperative Nachbehandlung}

Postoperativ ist nach perkutaner Schraubenosteosynthese oft keine Immobilisation erforderlich. Je nach der intraoperativ erreichten Stabilität und dem Patientenprofil kann eine Ruhigstellung von wenigen Tagen bis zu 2 Wochen sinnvoll erscheinen und wird entsprechend durch den Operateur festgelegt.

Die Belastung sollte nicht vor Ablauf von 6 Wochen und erst nach Vorliegen radiologischer Zeichen der knöchernen Durchbauung in den 3 Standardebenen der Skaphoiddiagnostik (pa, seitlich und Stecher) aufgenommen werden.

Sind diese nicht eindeutig, empfiehlt sich eine Kontrolle der Frakturheilung durch Computertomografie, wiederum in zusätzlicher schräg-sagittaler Schnittebene in der Längsachse des Skaphoids.

\section{Literatur}

${ }^{1}$ Baumeister HH, Greinemann H. Zur konservativen Behandlung des Skaphoidbruches der Handwurzel. Unfallchirurg 1989; 92: 175-179

2 Bond CD, Shin AY, McBride MTet al. Percutaneous screw fixation or cast immobilization for nondisplaced scaphoid fractures. J Bone Joint Surg 2001; 83:483-483

${ }^{3}$ Buijze GA, Goslings JC, Rhemrev SJ et al. Cast immobilization with and without immobilization of the thumb for nondisplaced and minimally displaced scaphoid waist fractures: a multicenter, randomized, controlled trial. J Hand Surg 2014; 39: 621-627

${ }^{4}$ Clay NR, Dias JJ, Costigan, PS et al. Need the thumb be immobilised in scaphoid fractures? A randomised prospective trial. J Bone Joint Surg Br 1991; 73: 828-832

${ }^{5}$ Herbert TJ, Fisher WE. Management of the fractured scaphoid using a new bone screw. J Bone Joint Surg Br 1984; 66:114-123
${ }^{6}$ Inoue G Shionoya K. Herbert screw fixation by limited access for acute fractures of the scaphoid. J Bone Joint Surg Br 1997; 79: 418-421 7 Jeon $\mathrm{IH}$, Micic ID, Oh CW et al. Percutaneous screw fixation for scaphoid fracture: a comparison between the dorsal and the volar approaches. J Hand Surgery 2009; 34: 228-236

8 Krimmer H. Skaphoidfraktur - Diagnostik und Therapie - aktueller Stand. Obere Extremität 2010; 5: 98-105

${ }^{9}$ Krimmer H, Schmitt $R$ Herbert T. Skaphoidfrakturen - Diagnostik, Klassifikation und Therapie. Unfallchirurg 2000; 103: 812-819

10 McQueen MM, Gelbke MK, Wakefield A. Percutaneous screw fixation versus conservative treatment for fractures of the waist of the scaphoid a prospective randomised study. J Bone Joint Surg Br; 90:66-71

11 Modi CS, Nancoo T, Powers D. Operative versus nonoperative treatment of acute undisplaced and minimally displaced scaphoid waist fractures - a systematic review. Injury 2009; 40, 268-273

12 Sauerbier M Müller M. Skaphoidfrakturen: Diagnostik, Zugangswege, Komplikationen. Zentralbl Chir 2007; 132: W42-W53

13 Schaefer M, Siebert HR. Die Skaphoidfraktur Unfallchirurg 2002; 105: 540-553

14 Stecher WR. Roentgenography of the carpal navicular bone. Am J Roentgenol 1937; 37: 704-705

15 Towfigh H, Hierner R, Langer M et al. Handchirurgie (Vol. 2). Berlin: Springer; 2011

\section{Dr. Benjamin Ziegler}

Assistenzarzt in Weiterbildung

Dr. Berthold Bickert

Leitender Oberarzt

Prof. Dr. Ulrich Kneser

Klinikdirektor

Klinik für Hand-, Plastische und

Rekonstruktive Chirurgie -

Schwerbrandverletztenzentrum

BG Unfallklinik Ludwigshafen am

Rhein

Ludwig-Guttmann-Straße 13

67071 Ludwigshafen

benjamin.ziegler@bgu-ludwigshafen.de 